\title{
Predicting Clinical Deterioration in Hospitals
}

\author{
$1^{\text {st }}$ Laleh Jalali \\ Hitachi America, Ltd. \\ Santa Clara, CA, USA \\ Laleh.Jalali@hal.hitachi.com
}

\author{
$2^{\text {nd }}$ Hsiu-Khuern Tang \\ Hitachi America, Ltd. \\ Santa Clara, CA, USA \\ Hsiu-Khuern.Tang@hal.hitachi.com
}

\author{
$3^{\text {rd }}$ Richard H. Goldstein \\ RGI Informatics, LLC. \\ Cornwallville, NY, USA \\ rgoldstein@rgi-informatics.com
}

$4^{\text {th }}$ Joaquín Álvarez Rodríguez
Department of Critical Care
Fuenlabrada University Hospital
Fuenlabrada, Madrid, Spain
joaquin.alvarez@ salud.madrid.org

\begin{abstract}
Responding rapidly to a patient who is demonstrating signs of imminent clinical deterioration is a basic tenet of patient care. This gave rise to a patient safety intervention philosophy known as a Rapid Response System (RRS), whereby a patient who meets a pre-determined set of criteria for imminent clinical deterioration is immediately assessed and treated, with the goal of mitigating the deterioration and preventing intensive care unit (ICU) transfer, cardiac arrest, or death. While RRSs have been widely adopted, multiple systematic reviews have failed to find evidence of their effectiveness. Typically, RRS criteria are simple, expert (consensus) defined rules that identify significant physiologic abnormalities or are based on clinical observation.

If one can find a pattern in the patient's data earlier than the onset of the physiologic derangement manifest in the current criteria, intervention strategies might be more effective. In this paper, we apply machine learning to electronic medical records (EMR) to infer if patients are at risk for clinical deterioration. Our models are more sensitive and offer greater advance prediction time compared with existing rule-based methods that are currently utilized in hospitals.
\end{abstract}

Our results warrant further testing in the field; if successful, hospitals can integrate our approach into their existing IT systems and use the alerts generated by the model to prevent ICU transfer, cardiac arrest, or death, or to reduce the ICU length of stay.

Index Terms-Healthcare Big Data Analytics, Patient Safety, Clinical Deterioration, Machine Learning

\section{INTRODUCTION}

More than 5.7 million patients are admitted annually to intensive care units (ICUs) in the United States [10], with an estimated cost ranging from 121 to 262 billion dollars a year [16] and a mortality rate of about $11 \%$ [15]. Unplanned admissions to the ICU are accompanied by an increase inhospital mortality rate and length of stay. Patients with deteriorating conditions often manifest abnormalities in physiological signals and laboratory test results before becoming unstable. The belief that rapid intervention in response to warning signs might provide a better outcome for these patients gave rise to a patient safety intervention philosophy known as a Rapid Response System (RRS). RRS use a pre-determined set of criteria to identify patients at risk of imminent clinical deterioration for immediate assessment and treatment. However,

978-1-7281-6251-5/20/\$31.00 @2020 IEEE systematic study of these rule-based systems has failed to show better outcomes as measured by a decrease in ICU transfers or deaths. There are a number of reasons that the current RRS are inadequate: 1) they are not sensitive enough, 2) they don't provide sufficient lead time for medical intervention, 3) they do not continuously monitor patients, or 4) they fail to systematically monitor all patients [2].

A comprehensive, automated, timely, and accurate approach to identifying patients who are at risk for clinical deterioration could improve RRS intervention outcomes. One approach is to apply machine learning on vast historical electronic medical records (EMR) data to create state-of-the-art systems for detecting clinical deterioration; if these AI-based systems can detect patients at risk earlier and more accurately (more sensitive, no less specific), this would allow hospitals to do more timely interventions, and potentially reduce mortality and improve prognosis.

In this study, our goal is to create an automated prediction model based on real-time electronic medical records to identify patients at high risk of clinical deterioration. We use unplanned ICU transfers as a proxy for clinical deterioration. We leverage thousands of predictor variables from EMR data, rather than dozens as is common in the current RRS. We hypothesized that such complex models provide better accuracy at longer lead time, providing more time and opportunity for clinicians to act to reverse deterioration. We compare our model with the Spanish ICU without walls (ICUWW) method [1], which has been used at the same hospitals.

\section{Conceptual Architecture}

Figure 1 shows a conceptual architecture for building and deploying our machine learning clinical deterioration model.

Data ingestion and preparation involves extracting data from EMR system, cleaning data, and unifying different coding standards. In practice, differences among hospitals can be substantial, making it difficult to implement and deploy an effective system across multiple hospitals. The adaptation of common coding standards by hospitals, such as LOINC for lab tests and fluids in/out, International Classification of Disease (ICD) for diagnoses and procedures, and ATC for medications, 


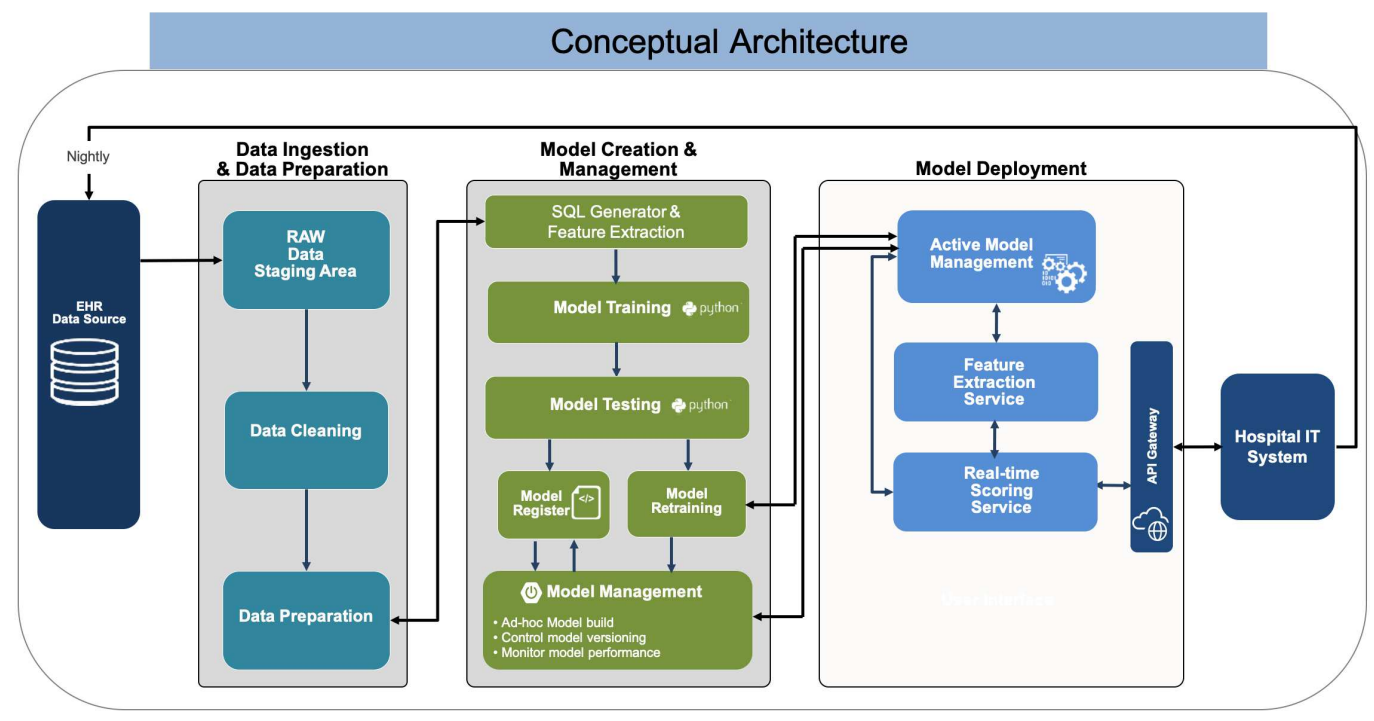

Fig. 1. A conceptual architecture for building and deploying machine learning solutions and big data algorithms in hospitals.

has helped to improve the usability of EMR data. Despite this, hospitals may use different standards for the same data, such as NDC and ATC for medications, or transition to a newer version of a standard, such as from ICD-9 to ICD-10. A robust system has to deal with this heterogeneity before building machine learning models. Moreover, since many EMR systems are still created and updated manually, there is room for human error and data quality issues. A proper data cleaning pipeline includes preparing data for analysis by removing or modifying data that is incorrect, incomplete, irrelevant, duplicated, or improperly formatted.

Model creation and management involves a feature extraction and a SQL generation tool that prepares data for model building phase. Extracted features and the SQL generation tool are explained in details in section

Integrating machine learning solutions and big data algorithms to the current hospital IT system is a challenging task. A closed loop architecture indicates how predictive data shows up in clinical workflows. To integrate predictive models into different EMR systems at the point of care, we can use application program interface (API) to retrieve realtime scoring predictions. As soon as new data is available for a patient, patient information along with the new data are submitted to an API Gateway. API Gateway makes a call to the scoring service and generates a prediction score. Model deployment component is not currently addressed in our machine learning solution. It will be implemented for operationalizing the solution in future.

\section{Research Method}

In this section, we give an overview of the data and define the prediction target. We formulate the real-time prediction of the target from the streaming EMR data as a classification problem and describe the features extracted for model learning. We also describe the model evaluation metrics and our modeling approach.

\section{A. Data}

The study population consists of about 200,000 admissions from three hospitals over a period of about 5 years. If a patient is admitted to the hospital from the Emergency Department (ED), the ED stay is not part of the study population. For this study, the goal is to predict unplanned ICU transfers, which after consulting with physicians at the department of critical care we use as a proxy for clinical deterioration.

Cases in our study involved unplanned transfers from an inpatient ward to the ICU. If a patient experienced more than one ICU transfer within the same hospital admission, we only consider the first unplanned ICU transfer since the subsequent ICU transfers are considered as ICU readmission problem. The cases are identified using admission, discharge, and transfer records by selecting patients who are admitted to a general ward followed by a transfer to the ICU. We exclude ICU stays that occur at the start of an admission. This indicates a scenario where a patient is transferred to ICU from Emergency Department. Hence, we do not have access to relevant EMR data to make timely predictions for this scenario. We also exclude ICU transfers from an operating room bed or a recovery bed since those patients did not actually experience clinical deterioration, but were transferred routinely to the ICU after a surgery and those transfers are expected. Moreover, we exclude any neonatal patient data and subsequently any transfers to the neonatal intensive care unit.

Our prediction target is the first unplanned ICU transfer. Table \shows the categories of patient data provided for each admission. Patient demographics include age and gender. The admission, discharge, and transfer history records the transfers 
of the patient from one ward to another. Fluids in/out, lab tests, and vital signs are collected over irregularly spaced time intervals. For all the measurements, we have access to the times at which each value was gathered. Examples of fluids in/out are oral intake, IV intake, urine output, wound drain, and 8-hour balance, etc. that are collected two to four times a day from a patient. Some of the standard vital signs are the patient's heart rate, respiration rate, glucose level, oxygen level (SpO2), and systolic and diastolic blood pressure. Vital sign measurements are taken more frequently, ranging from 1 to 4 hours, from a patient compared to fluids in/out. In addition, there are more than 300 different lab items in the data.

TABLE I

EMR DATA CATEGORIES

Patient demographics
Admission, discharge, and transfer history
Fluids in/out
Vital signs
Lab tests
Medication orders and administration
Medications on admission
Diagnoses
Procedures

\section{B. Problem Formulation}

We seek to learn a model for the risk of unplanned ICU transfer from historical patient data. For each admission, we select a set of times at which to calculate the risk scores, based on the arrival timestamps of new or updated patient data, such as fluids in/out measurements, vital signs, lab test results, and medications administered. At each selected time, we calculate a set of features known about the admission at that time.

To illustrate the process, Figure 2 shows two streams of data for an admission. For each fluids measurement or vital sign, the corresponding timestamp and value is added to a patient master table. Features for model learning are then calculated from the accumulated data at each timestamp.

\section{Feature Extraction}

Table II shows some of the features that we created, including counts, averages, most recent values, and trends, calculated over various time windows. The Charlson Comorbidity Index is a method of categorizing comorbidities of patients based on the ICD diagnosis codes [17]. Comorbidity means more than one disease or condition is present in the same person at the same time. Charlson comorbidity contains 17 categories, each comorbidity category has an associated weight (from 1 to 6), based on the adjusted risk of mortality or resource use, and the sum of all the weights results in a single comorbidity score for a patient. A score of zero indicates that no comorbidities were found. The higher the score, the more likely the predicted outcome will result in mortality or higher resource use. The Elixhauser Comorbidity Index is another method for measuring patient comorbidity based on ICD diagnosis codes. The original Index was developed with 30 categories [18]. Walraven et al. [19] developed a weighting algorithm based on the association between comorbidity and death, in order to produce an overall score for the Elixhauser Index.

The data on diagnoses, procedures, and medications on admission are only known after discharge; hence, we can only use such data from a patient's past admissions. We calculated Charlson and Elixhauser comorbidity indexes from previous admissions of the patient, within one year.

Some data cleaning was done to improve the quality and interpretability of the features. As examples: 1) we extracted the numeric lab values from the underlying text representation, and 2) we rounded the medication codes to the fourth level of the ATC (Anatomical Therapeutic Chemical) hierarchy, to reduce the number of distinct medications considered.

The combined data for all three hospitals is a matrix with about 10 million rows and 500 columns. The rows arise from the approximately 200,000 admissions and all the times within those admissions, and the columns correspond to the features and the prediction target, which has value 1 or 0 according to whether the admission contains an unplanned ICU transfer or not.

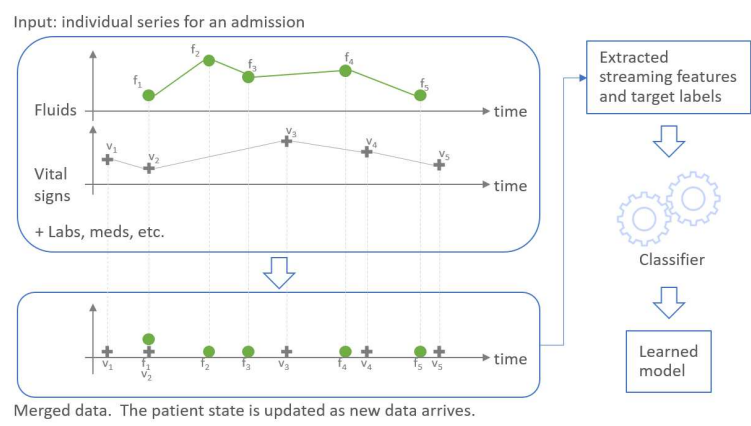

Fig. 2. We merge individual data series into a training dataset, which represents the dynamic state of a patient during the admission. Features are created from the patient state and used to learn a model for predicting unplanned ICU transfers.

During deployment, the model needs to be retrained periodically to incorporate the latest data and new hospitals that are onboarded to the system. For maximum flexibility, we allow the features for model learning to reside in different tables; for example, they might be organized by hospital and category (Table I). We developed a simple language for specifying the features to include for modeling, their source tables, and their key columns. We wrote a parser for this language that generates a SQL statement for merging those features into a single table.

The code in Listing 1 shows an example of a generated SQL statement. Our parser supports the following attributes:

- Renaming (line 4)

- Inline expressions to define new features (line 7) 
TABLE II

EXAMPLES OF FEATURES FOR MODEL LEARNING

\begin{tabular}{|l|l|}
\hline Category & Features \\
\hline Demographics & $\begin{array}{l}\text { Age } \\
\text { Gender }\end{array}$ \\
\hline \multirow{2}{*}{ Usage recency and frequency } & $\begin{array}{l}\text { Number of previous admissions } \\
\text { Previous length of stay } \\
\text { Previous discharge disposition } \\
\text { Days since last discharge } \\
\text { Number of admissions in the past } x \text { months, } x \in\{1,6,12\} \\
\text { Trend of the number of lab tests }\end{array}$ \\
\hline Transfer history & $\begin{array}{l}\text { Ward at admission } \\
\text { Most recent ward }\end{array}$ \\
\hline $\begin{array}{l}\text { Fluids in/out: } \\
\text { oral intake, IV intake, } \\
\text { urine output, 8-hour balance, etc. }\end{array}$ & $\begin{array}{l}\text { Most recent values } \\
\text { Count of the measurements in the past } y \text { days, } y \in\{1,3,5,7\} \\
\text { Average of the measurements in the past } y \text { days } \\
\text { Trend of 8-hour fluid balance } \\
\text { Trend of urine output }\end{array}$ \\
\hline $\begin{array}{l}\text { Vital signs: } \\
\text { heart rate, respiration rate, oxygen level (SpO2), } \\
\text { systolic and diastolic blood pressure, glucose, etc. }\end{array}$ & $\begin{array}{l}\text { Most recent values } \\
\text { Count of the measurements in the past } y \text { days } \\
\text { Average of the measurements in the past } y \text { days }\end{array}$ \\
\hline & $\begin{array}{l}\text { Most recent value of each lab test } \\
\text { Count of each lab test since admission } \\
\text { Count of each lab test in the past } y \text { days } \\
\text { Average of lab test values in the past } z \text { weeks, } z \in\{1,2,3,4\} \\
\text { Minimum of lab test values in the past } z \text { weeks } \\
\text { Maximum of lab test values in the past } z \text { weeks }\end{array}$ \\
\hline $\begin{array}{l}\text { Lab items: } \\
\text { more than 300 lab items }\end{array}$ & $\begin{array}{l}\text { Modified Charlson comorbidity in the past year } \\
\text { Modified Elixhauser comorbidity in the past year }\end{array}$ \\
\hline $\begin{array}{l}\text { Number of medications administered in the past } y \text { days. } \\
\text { Mast diagnoses }\end{array}$ &
\end{tabular}

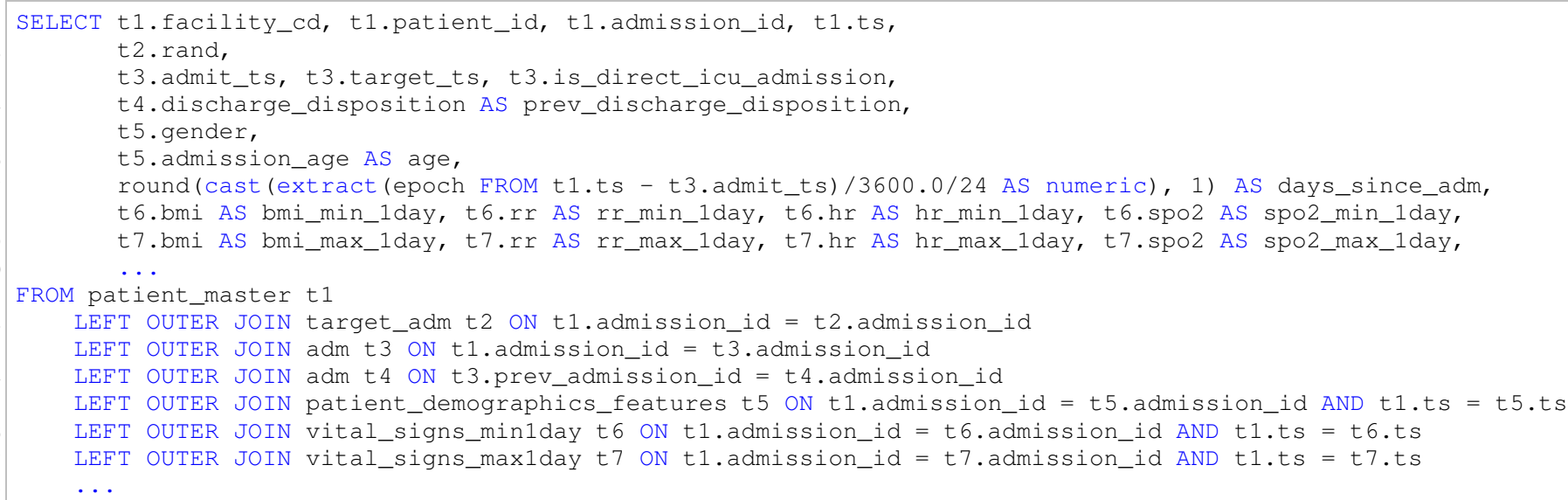

Listing 1. Automatically generated SQL to merge features

- Wildcards to select multiple features from a table (lines 8-9)

- Custom join columns, including self-joins (lines 13-14)

Our tool makes it feasible for the end user to select features for retraining the model without knowing the underlying details.

\section{Machine Learning Method}

The learned model can be used to calculate the scores for a patient over time, based on the latest data available. Figure 3 shows an example of the predicted scores for a single admission. When the score crosses a pre-specified threshold for the first time, an alert can be generated to alert the medical staff to assess the patient. The threshold corresponds to a particular sensitivity and specificity of this early warning system.

We evaluate model performance at the admission level. Each admission is either correctly or wrong classified: a false positive occurs when an alert is generated for an admission with no unplanned ICU transfer, and a false negative occurs when no alert is generated for an admission with an unplanned ICU transfer. From these, we can calculate the sensitivity (ratio of true positives to actual positives), specificity (ratio of true negatives to actual negatives), and AUC of the model. The AUC is equal to the area under the sensitivity-specificity 


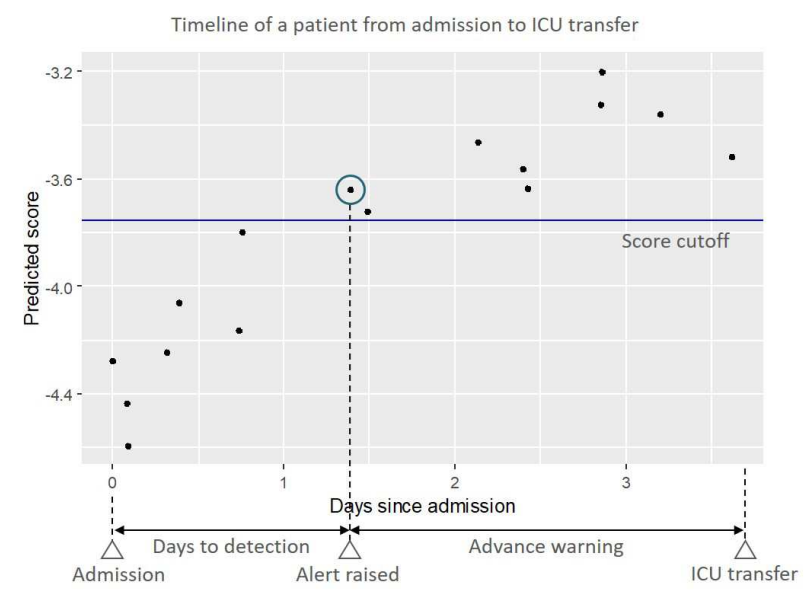

Fig. 3. The predicted scores at various times within an admission are shown. A score is calculated when there is updated data for that admission. The blue line indicates the score threshold. An alert can be generated when the threshold is crossed for the first time.

curve as the score threshold varies. For true positives, we also measure the advance warning, which is the time between the first alert and the target event.

We selected $80 \%$ of the admissions at random for model training and validation. The remaining $20 \%$ formed the test set for model evaluation. We learned a separate model for each hospital, using its own data. We treat our problem as one of binary classification, where for each admission and timestamp, we seek to predict whether that admission had an unplanned ICU transfer.

We focused on tree-based models because they have natural ways of dealing with missing data, which is prevalent in our data. For example, of the more than 200 possible lab tests, most are rarely administered, leading to lots of missing values for those tests. We decided to choose XGBoost because it is modern library and readily supports a custom evaluation metric, which we need.

We used the training and validation data to select the following model hyperparameters: 1) the sampling rate of negative cases, 2) the learning rate, 3) the maximum depth of the trees, 4) the sampling rate of features used to construct each tree, and 5) the number of cases in a node below which no further splitting is done.

For an unbalanced dataset like ours, where $99 \%$ of the admissions do not have an unplanned ICU transfer, sampling these negative cases is a way to speed up model learning without sacrificing model performance. Based on our experiments, using about $1 / 4$ of the negative admissions and all the positive admissions works well for model learning. The other four hyperparameters come from XGBoost; we selected them via a trial-and-error approach based on the experience of XGBoost practitioners.

For a given choice of the above parameters, we selected the number of boosting iterations by 5 -fold cross-validation to maximize the admission-level AUC. Since the predictions are made for each admission and timestamp, the default AUC is calculated by comparing the predicted score $\hat{y}(a, t)$ for each admission $a$ at various timestamps $t$ with the actual class $y(a)$ of that admission. However, for our purpose, each admission is either correctly classified or not, so it is better to use the AUC obtained by comparing the maximum predicted score $\max _{t} \hat{y}(a, t)$ within an admission with the actual class $y(a)$. Figure 4 illustrates how using the default AUC may lead to a suboptimal choice for the number of boosting iterations.

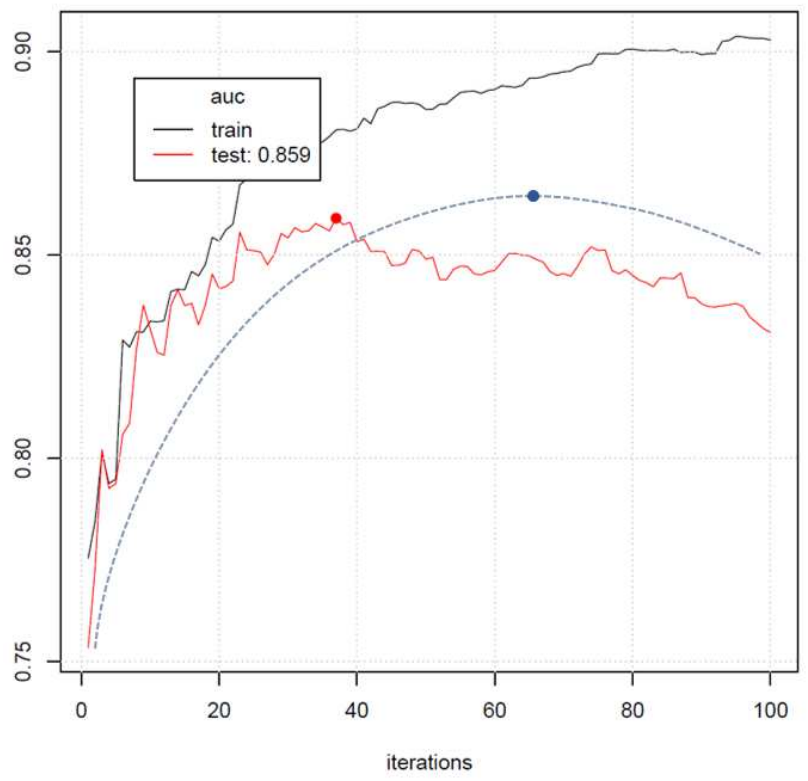

Fig. 4. An example showing how the number of boosting iterations is selected to maximize the AUC. The default AUC (blue dashed line) reaches its maximum between 60 and 70 iterations, a region where the desired admissionlevel AUC (red line) is suboptimal.

\section{RESUlts}

In this section, we compare our models with the ICU without walls (ICUWW) method [1], which was used at the same hospitals. Under this method, an alert is raised if any of the lab results shown in Table [II] are observed. We implemented these criteria for all admissions in our data, using the same set of timestamps described earlier.

Table IV] shows the performance of our models and of ICUWW. For each method, we report its sensitivity, specificity, AUC, the median advance warning for true positives, and the mean number of admissions per day with an alert. For our models, these metrics were calculated on the $20 \%$ test set that was not used for model learning.

The ICUWW method has high specificity but low sensitivity; its average sensitivity over the three hospitals is only $37 \%$. This is perhaps unsurprising: the 30 "track and trigger" scoring systems reviewed in [11] have a median sensitivity of $24 \%$ and a median specificity of $92 \%$.

In Table IV, two settings of the score threshold are shown for each model: one with the same specificity as ICUWW and one with a specificity of $75 \%$. At the same specificity, our 
TABLE III

ICU WITHOUT WALLS BENCHMARK

\begin{tabular}{|l|l|l|l|}
\hline Lab Item & Lab Text Description & LOINC Code & Criteria \\
\hline Troponin I & Troponin I.cardiac [Mass/volume] in Serum or Plasma & $10839-9$ & $>0.3 \mathrm{ug} / \mathrm{L}$ \\
\hline Arterial $\mathrm{pH}$ & pH of Arterial blood & $2744-1$ & $<7.30$ \\
\hline Arterial $\mathrm{pCO} \mathrm{CO}_{2}$ & Carbon dioxide [Partial pressure] in Arterial blood & $2019-8$ & $>60 \mathrm{mmHg}$ \\
\hline Platelets & Platelets [\#/volume] in Blood by Automated count & $777-3$ & $<100,000 / \mathrm{uL}$ \\
\hline Lactate & Lactate in Serum/Plasma & $2524-7$ & $>3 \mathrm{mmol} / \mathrm{L}$ \\
\hline
\end{tabular}

models are more sensitive, have the same or greater advance warning, and raise a similar number of alerts. The models also have AUCs above 0.85, compared with 0.57-0.69 for ICUWW (these are independent of the threshold).

Even though the sensitivities are improved, they are still too low to be useful. If the model specificities are lowered to $75 \%$, the sensitivities are increased substantially, to about $80-85 \%$, at the cost of raising about 3 times as many alerts. Figure 5 shows the sensitivities and specificities that can be attained by our models by varying the score threshold. Our models also compare favorably with mammography, a routine screening test, which has a sensitivity of $67.8 \%$ and a specificity of $75 \%$.

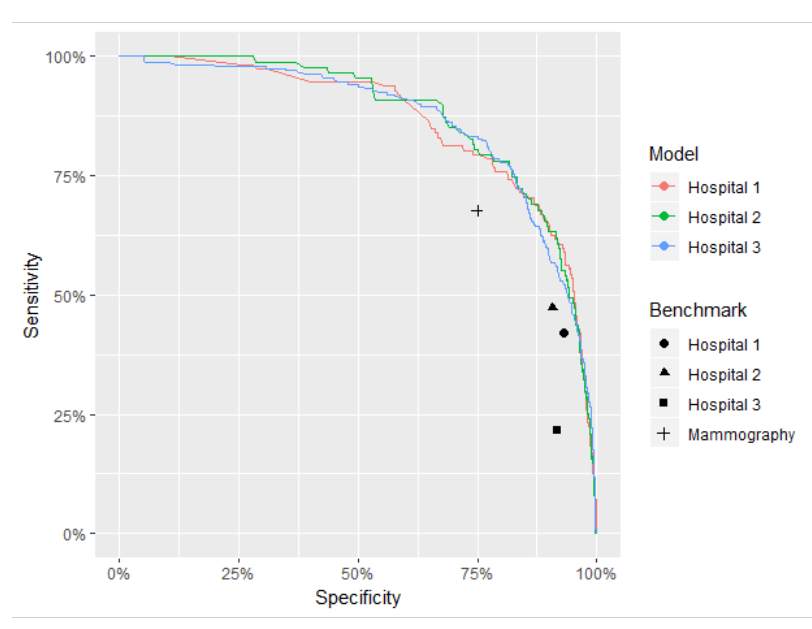

Fig. 5. The colored lines show the trade-off between sensitivity and specificity for the three hospitals. The four marked points show the performance of the ICUWW benchmark and of mammography screening tests.

We make a final observation from Table IV] the median advance warning for hospital 3 is much shorter than the other two hospitals. We found that this is because hospital 3 sends a significantly larger fraction of its patients to the ICU, and does so sooner. If we omit admissions where the ICU transfer occurred within 24 hours of the patient being admitted, the median advance warning for hospital 3 increases to 1.5 days for ICUWW and 2.8 days for the model with $75 \%$ specificity. The average for the three hospitals becomes 2.9 days for ICUWW and 3.6 days for the model.

Figure 6 shows the actual unplanned ICU transfer rates within each predicted score quintile of the test set. The first quintile comprises admissions with scores in the bottom fifth of all scores, the second quintile comprises the next fifth, and so on. We observe a strong correlation between the predicted score and the ICU transfer rate. This shows that our models are well calibrated.

\section{A. Explaining the model}

Feature importance and feature attribution are two concepts that are useful for explaining a model. For tree-based models, there are appealing definitions for operationalizing these concepts, as well as efficient calculation methods.

Feature importance measures how useful each feature is for constructing the model or for determining the model predictions. Suppose the model depends on the input features $x_{1}, x_{2}$, $\ldots, x_{p}$. For a decision tree that is constructed by recursively splitting the training data on a single feature to maximize the decrease in some impurity measure (such as the Gini index), one common definition of the importance of a feature $x_{i}$ is the mean decrease in impurity over all splits on $x_{i}$ weighted by the number of training examples in those splits. For a model that is a sum or average of decision trees, we would simply take the sum or average of the per-tree feature importance values.

For XGBoost, the splits are selected to minimize a different objective function based on a loss function, and a similar definition for feature importance can be made.

In feature attribution, we seek to allocate an individual model prediction $\hat{y}$ to the features: $\hat{y}=$ constant $+\phi_{1}+\cdots+$ $\phi_{p}$. Recently, an attribution method based on Shapley values has gained popularity because of its nice theoretical properties and the availability of an efficient software implementation for tree-based models ( [9]). The contributions can be positive or negative, and for a feature $x_{i}$, its total absolute Shapley contribution $\sum\left|\phi_{i}\right|$ over the training set may be used as another definition of the importance of $x_{i}$.

We applied the above techniques to our three models, one for each hospital. From our analysis, age is the most important feature in all three models. Some vitals signs and lab tests are also important, although the specific ranking of these features vary across the hospitals. For vital signs, heart rate, $\mathrm{SpO}$, and $B M I$ are generally important. One surprising discovery is that certain rarely administered lab tests are important. They would likely not be discovered if we had applied a sparsity criterion for feature selection prior to model fitting. Table $\mathrm{V}$ shows the list of 30 important features, without any specific order of importance, across all three models. 
TABLE IV

PERFORMANCE OF THE MODELS AND THE ICU WITHOUT WALLS BENCHMARK. TWO MODELS ARE SHOWN FOR EACH HOSPITAL, DIFFERING ONLY IN THEIR SCORE THRESHOLDS: MODEL A HAS THE SAME SPECIFICITY AS THE BENCHMARK AND MODEL B HAS A SPECIFICITY OF $75 \%$.

\begin{tabular}{l|l|r|r|r|l|c}
\hline Hospital & Method & Sensitivity & Specificity & AUC & $\begin{array}{l}\text { Advance } \\
\text { warning }\end{array}$ & $\begin{array}{l}\text { Admissions per } \\
\text { day with alerts }\end{array}$ \\
\hline 1 & ICUWW & $42.0 \%$ & $93.1 \%$ & 0.676 & 2.4 days & 2.9 \\
& Model A & $58.9 \%$ & $93.1 \%$ & 0.862 & 2.4 days & 3.0 \\
& Model B & $79.5 \%$ & $75.0 \%$ & 0.862 & 2.7 days & 11 \\
\hline 2 & ICUWW & $47.3 \%$ & $90.7 \%$ & 0.690 & 2.1 days & 3.8 \\
& Model A & $63.2 \%$ & $90.7 \%$ & 0.873 & 3.4 days & 3.8 \\
& Model B & $80.5 \%$ & $75.0 \%$ & 0.873 & 4.0 days & 10 \\
\hline 3 & ICUWW & $21.6 \%$ & $91.7 \%$ & 0.566 & 0.3 days & 2.4 \\
& Model A & $55.9 \%$ & $91.7 \%$ & 0.863 & 0.3 days & 2.6 \\
& Model B & $85.3 \%$ & $75.0 \%$ & 0.863 & 0.4 days & 7.3 \\
\hline
\end{tabular}
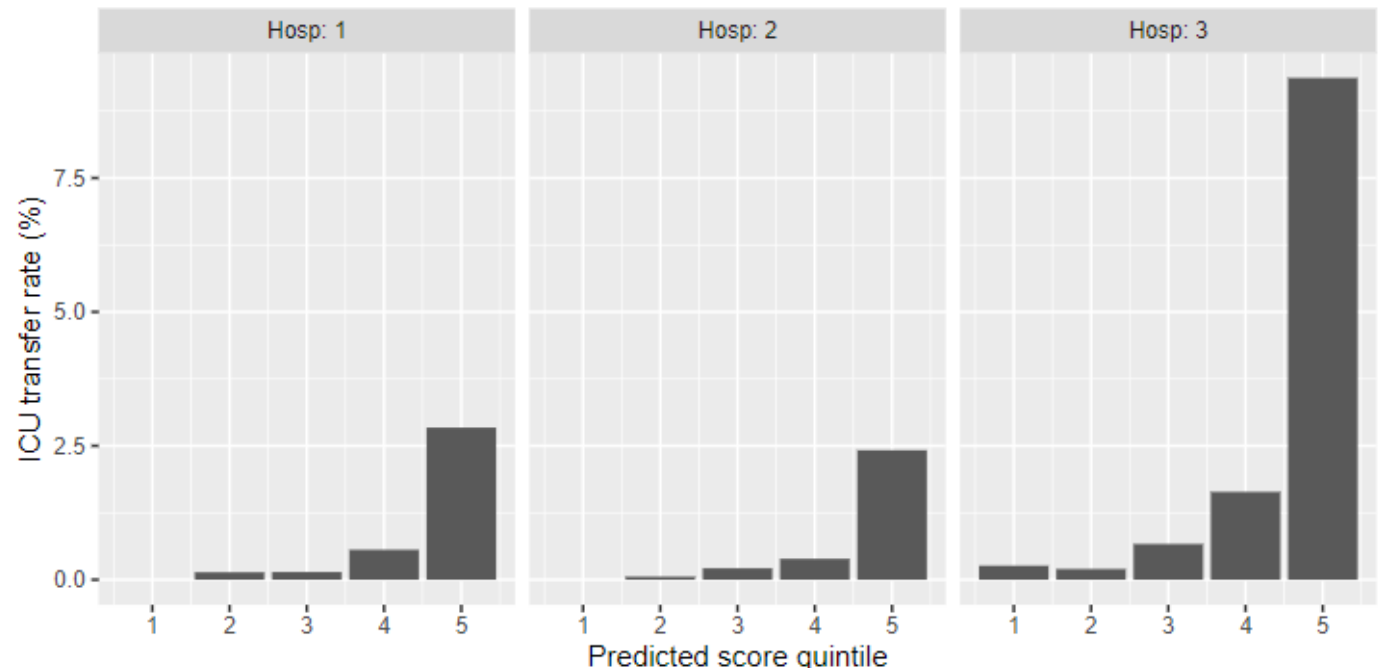

Fig. 6. The test set admissions for each hospital are divided into approximately equal-sized groups 1-5 in ascending order of their predicted scores. The bars show the rates of ICU transfers for these groups.

\section{RELATED WORK}

The Joint Commission (https://www.jointcommission.org/) is an independent, not-for-profit organization that aims to improve health care for the public in collaboration with other stakeholders. A panel of patient safety experts advises the Joint Commission on a set of patient safety goals called the National Patient Safety Goals (NPSGs). NPSG requires hospitals to implement systems to enable intervention from healthcare staff when a patient's condition is worsening, which has led to the Rapid Response System (RRS) intervention philosophy. Some studies have shown that the implementation of RRS slightly reduces cardiac arrest in general ward patients but does not reduce overall hospital mortality [14].

The Modified Early Warning System (MEWS) [12] is a widely used physiologic scoring system for warning about clinical deterioration. It is based on the patient's respiratory rate, $\mathrm{SpO}$, temperature, systolic blood pressure, heart rate, and level of consciousness. MEWS typically offers an advance warning of deterioration of 6 to 8 hours. Our benchmark ICUWW is another scoring system; it uses five lab test results. Recent systematic reviews have demonstrated that trigger alarms only marginally improve outcomes while substantially increasing physician and nursing workloads [22]. Moreover, these methods respond to triggering events that may not signal a truly deteriorating patient, they suffer from high rates of false alarms (70-95\%), which results in alarm fatigue and inappropriate resource utilization.

Although such rule-based systems have been widely adopted by hospitals, multiple systematic reviews have failed to find evidence of their effectiveness. Typical calling criteria are based on physiologic measurements, nurse assessments, and sometimes lab tests. Alvarez et al. [2] trained a logistic regression model using comprehensive EMR data to identify patients at high risk of out-of-ICU resuscitation events and death. They found fourteen important predictors, including age, oxygenation, diastolic blood pressure, arterial blood gas, lab test results, emergent orders, and assignment to a high-risk floor. Their automated model outperformed MEWS, obtaining a sensitivity of $42.2 \%$, a specificity of $91.3 \%$, and an advance warning of 15.9 hours. Their risk scores are not calculated in real-time; rather, data from the previous day are used to determine the score for the current day. In contrast, our 
TABLE V

THE LIST OF IMPORTANT FEATURES ACROSS THREE HOSPITAL MODELS. THE LIST IS COMPILED BY CONSIDERING TOP 15 FEATURES FROM EACH MODEL.

\begin{tabular}{|c|c|c|c|c|}
\hline \multicolumn{5}{|c|}{ Classification of important features } \\
\hline Lab tests & Vital signs & Fluids in/out & Service codes & $\begin{array}{l}\text { Demographics and } \\
\text { Usage recency and } \\
\text { frequency }\end{array}$ \\
\hline $\begin{array}{l}\text { - Lymphocytes in Blood } \\
\text { - Base excess in Blood } \\
\text { - Urea nitrogen in Serum or Plasma } \\
\text { - Cholesterol in Serum or Plasma } \\
\text { - Segmented neutrophils/100 leukocytes in Blood } \\
\text { - Triglyceride in Serum or Plasma } \\
\text { - Oxygen saturation in Arterial blood } \\
\text { - Thyrotropin receptor Ab in Serum } \\
\text { - Cancer Ag 19-9 in Serum or Plasma } \\
\text { - Osmolality of Serum or Plasma } \\
\text { - S100 calcium binding protein B in Serum } \\
\text { - Calcium corrected for albumin in Serum or } \\
\text { Plasma } \\
\text { - S100 calcium binding protein B in Serum } \\
\text { Oxygen [Partial pressure] in Venous blood }\end{array}$ & $\begin{array}{l}\text { - Heart rate max 1day } \\
\text { - Heart rate mrv } \\
\text { - Heart rate avg } \\
\text { - Systolic b.p. avg } \\
\text { - Weight mrv } \\
\text { - BMI mrv } \\
\text { - SpO2 avg } \\
\text { - Respiratory rate mrv }\end{array}$ & $\begin{array}{l}\text { - IV fluid max 1day } \\
\text { - 8-hour fluid balance } \\
\text { min 1day }\end{array}$ & $\begin{array}{l}\text { - Digestive system is } \\
\text { most recent service } \\
\text { - Admitted to General } \\
\text { Surgery \& Digestive } \\
\text { System }\end{array}$ & $\begin{array}{l}\text { - Age } \\
\text { - Previous length } \\
\text { of stay } \\
\text { - Days since prev. } \\
\text { discharge } \\
\text { - Modified } \\
\text { Elixhauser } \\
\text { index }\end{array}$ \\
\hline
\end{tabular}

formulation mimics the real-time streaming of EMR data into a prediction model.

In many cases, the patients' conditions deteriorate quickly and suffers a cardiac arrest or death outside the ICU. Some studies consider out-of-ICU mortality as a secondary outcome and aim to find factors associated with it. Wengerter et al. [13] evaluated Rothman Index variability for predicting rapid response team activation as the primary outcome and inhospital mortality as a secondary outcome. The Rothman Index incorporates twenty-six data points from EMR, including vital signs, lab test results, cardiac rhythm, and nursing assessments. It is based on the contribution of each factor to a model for predicting one-year mortality, but the Rothman Index itself is not modeled to predict any particular outcome. The authors considered the Rothman Index standard deviation (RISD) and the maximum-minus-minimum Rothman Index (MMRI) over a 24-hour window as two measures of variability. For predicting rapid response team activation, RISD and MMRI have AUCs of 0.74 and 0.76 , sensitivities of $91.7 \%$ and $92.2 \%$, and specificities of $39.9 \%$ and $37.3 \%$, respectively. They found no association between these Rothman Index variability measures and in-hospital mortality.

Both MEWS and Rothman Index scores require manual entry by nurses and only consider small sets of data categories. Rothman Index only considers 26 data points and other work on predicting deterioration [20] [21] uses smaller feature sets than ours and make use of 21 predictor variables. In contrast, we have upwards of over thousands of predictor variables across six different types of EMR data categories (shown in Table 【). We believe our rich set of predictor variables not only improves the accuracy of our models but increases their robustness to missing data.

Another body of research targets ICU readmissions. ICU readmissions may be a more difficult target for machine learning than the first ICU transfer since the former involves patients who have been discharged from the ICU by a human expert (the physician) presumably after extensive tests and monitoring. Desautels et al. [4] learned a model to predict death and 48-hour ICU readmission when a patient is first discharged from the ICU. Their AdaBoost model containing 1,000 decision trees was trained on the MIMIC III dataset [7] and achieved an accuracy of 70\%, a sensitivity of 59\%, and a specificity of $66 \%$.

\section{DISCUSSION}

In this paper, we built machine learning models for three hospitals to predict unplanned transfers of patients to the ICU as a proxy for clinical deterioration outside the ICU. The models are tunable with a good trade-off possibility between sensitivity and specificity. Also, the models take into account comprehensive data from EMR records and achieve AUCs above 0.85 . When the specificity is set to $75 \%$, the model sensitivities are around $80-85 \%$, with greater advance warning compared to widely-used rule-based systems and other studies that apply predictive models.

With its higher sensitivity and earlier warnings, our approach has the potential to detect more patients with clinical deterioration as well as increase the chance of a successful intervention. The next steps are first to determine the performance of the model in a real-world clinical setting and then to see whether using the model in a Rapid Response System demonstrates improved outcomes.

In addition to learning a separate model for each hospital, we also tried learning a single model using the combined data from all three hospitals. The single-model results (not shown) are promising, with only a modest decrease in performance compared with the separate models. Having the same model work for multiple hospitals is important for both scalability and adoption, since it is impractical to create a separate model for each hospital that needs such a system - the cost of data collection and preparation alone will deter adoption. 
For a solution provider, the biggest challenge to operationalizing a model is scalability. The data from different hospitals are heterogeneous and need to be normalized before they can be merged to create a single model. For example, the three hospitals in our study use different names to describe their wards, which we mapped to a common set of the most frequent names. To scale out our approach, significant work is needed to define common representations for all the important EMR data elements.

In general, value co-creation is an important aspect of innovation in the healthcare industry. Before developing any AI solution, machine learning scientists and healthcare administrators should have a clear value model and continue to collaborate after the integration of the solution in the hospital's current IT system. In the case of our solution for predicting clinical deterioration, the biggest challenge for a hospital with an existing Rapid Response System is in transitioning from an intermittent system of patient monitoring to an always-on system using streaming data. Other issues that need to be addressed before operationalizing the solution are: 1) estimating the the efforts required to deal with hospital variations when we implement our system on a new hospital; 2) addressing model drift as data and clinical practice change over time; and 3 ) whether the value of the clinical improvements obtained by using the model outweigh the cost of implementing and maintaining the model.

In the healthcare analytics, there is a trade-off between a more generalized prediction model that inputs big data and global features and a more specific model with a targeted use case. The features that effectively characterize a condition are the same attributes that can train an accurate predictor. But if those features do not stand out above the background noise, then the predictor only finds the noise. For this reason, predictions that are focused on a specific clinical goal will always supersede a generic predictor in terms of accuracy and utility. Hence, the full power of clinical prediction is best realized when the computational question is carefully defined, specific variables are gathered, and a targeted need is met. Our study with the carefully defined goal and attentively characterized predictors that rooted in clinical knowledge of physicians is a good example to showcase the full power of clinical prediction in an important real-life application.

\section{REFERENCES}

[1] Álvarez, A.A., Pérez, I.T., Calderón, V.E., Gelbard, C.H., Urban, J.S., Grinspan, M.R., Ureña, M.G., Gabiña, I.S., Martín, T.M., Herranz, E.C. and Blázquez, M.D., 2013. ICU without walls project. Effect of the early detection of patients at risk. Medicina Intensiva (English Edition), 37(1), pp.12-18.

[2] Alvarez, C.A., Clark, C.A., Zhang, S., Halm, E.A., Shannon, J.J., Girod, C.E., Cooper, L. and Amarasingham, R., 2013. Predicting out of intensive care unit cardiopulmonary arrest or death using electronic medical record data. BMC medical informatics and decision making, 13(1), p.28.

[3] Chen, T. and Guestrin, C., 2016, August. Xgboost: A scalable tree boosting system. In Proceedings of the $22 \mathrm{nd}$ acm sigkdd international conference on knowledge discovery and data mining, pp. 785-794.
[4] Desautels, T., Das, R., Calvert, J., Trivedi, M., Summers, C., Wales, D.J. and Ercole, A., 2017. Prediction of early unplanned intensive care unit readmission in a UK tertiary care hospital: a cross-sectional machine learning approach. BMJ open, 7(9).

[5] Friedman, J.H. and Popescu, B.E., 2008. Predictive learning via rule ensembles. The Annals of Applied Statistics, 2(3), pp.916-954.

[6] Friedman, J.H., 2001. Greedy function approximation: a gradient boosting machine. Annals of statistics, pp.1189-1232.

[7] Johnson, A.E., Pollard, T.J., Shen, L., Li-Wei, H.L., Feng, M., Ghassemi, M., Moody, B., Szolovits, P., Celi, L.A. and Mark, R.G., 2016. MIMICIII, a freely accessible critical care database. Scientific data, 3(1), pp.1-9.

[8] Ke, G., Meng, Q., Finley, T., Wang, T., Chen, W., Ma, W., Ye, Q. and Liu, T.Y., 2017. Lightgbm: A highly efficient gradient boosting decision tree. In Advances in neural information processing systems, pp. 31463154.

[9] Lundberg, S.M., Erion, G.G. and Lee, S.I., 2018. Consistent individualized feature attribution for tree ensembles. arXiv preprint arXiv: 1802.03888

[10] Critical care statistics. http://www.sccm.org/Communications/Critical-Care-Statistics July 2019.

[11] Smith, G.B., Prytherch, D.R., Schmidt, P.E., Featherstone, P.I. and Higgins, B., 2008. A review, and performance evaluation, of singleparameter "track and trigger" systems. Resuscitation, 79(1), pp.11-21.

[12] Subbe, C.P., Kruger, M., Rutherford, P. and Gemmel, L., 2001. Validation of a modified Early Warning Score in medical admissions. Qjm, 94(10), pp.521-526.

[13] Wengerter, B.C., Pei, K.Y., Asuzu, D. and Davis, K.A., 2018. Rothman Index variability predicts clinical deterioration and rapid response activation. The American Journal of Surgery, 215(1), pp.37-41.

[14] Winters, B.D., Weaver, S.J., Pfoh, E.R., Yang, T., Pham, J.C. and Dy, S.M., 2013. Rapid-response systems as a patient safety strategy: a systematic review. Annals of internal medicine, 158(5_Part_2), pp.417425 .

[15] Zimmerman, J.E., Kramer, A.A. and Knaus, W.A., 2013. Changes in hospital mortality for United States intensive care unit admissions from 1988 to 2012. Critical care, 17(2), p.R81.

[16] Nates, J.L., Nunnally, M., Kleinpell, R., Blosser, S., Goldner, J., Birriel, B., Fowler, C.S., Byrum, D., Miles, W.S., Bailey, H. and Sprung, C.L., 2016. ICU admission, discharge, and triage guidelines: a framework to enhance clinical operations, development of institutional policies, and further research. Critical care medicine, 44(8), pp.1553-1602.

[17] Deyo, R.A., Cherkin, D.C. and Ciol, M.A., 1992. Adapting a clinical comorbidity index for use with ICD-9-CM administrative databases. Journal of clinical epidemiology, 45(6), pp.613-619.

[18] Elixhauser, A., Steiner, C., Harris, D.R. and Coffey, R.M., 1998. Comorbidity measures for use with administrative data. Medical care, pp.8-27.

[19] van Walraven, C., Austin, P.C., Jennings, A., Quan, H. and Forster, A.J., 2009. A modification of the Elixhauser comorbidity measures into a point system for hospital death using administrative data. Medical care, pp.626-633.

[20] Yoon, J., Alaa, M. A., Hu, S., and Van Der Schaar, M., 2013, ForecastICU: a prognostic decision support system for timely prediction of intensive care unit admission, Proceedings of the 33rd International Conference on International Conference on Machine Learning, Volume 48, pp.1680-1689.

[21] Alaa, M. A. and Van Der Schaar, M., 2018. A Hidden Absorbing SemiMarkov Model for Informatively Censored Temporal Data: Learning and Inference, Journal of Machine Learning Research, pp.1-62.

[22] Alam, N, Hobbelink, EL, van Tienhoven, AJ, van de Ven, PM, Jansma, EP, and Nanayakkara, 2014. PWB. The impact of the use of the early warning score (ews) on patient outcomes: a systematic review. Resuscitation, 85(5), pp.587-594. 